\title{
Presbifagia Una mirada a los procesos de alimentación y deglución en los adultos mayores
}

Presbyphagia: A look at feeding and swallowing processes in older adults

(c) $\frac{10(0)}{\mathrm{Br} N \mathrm{Na}}$
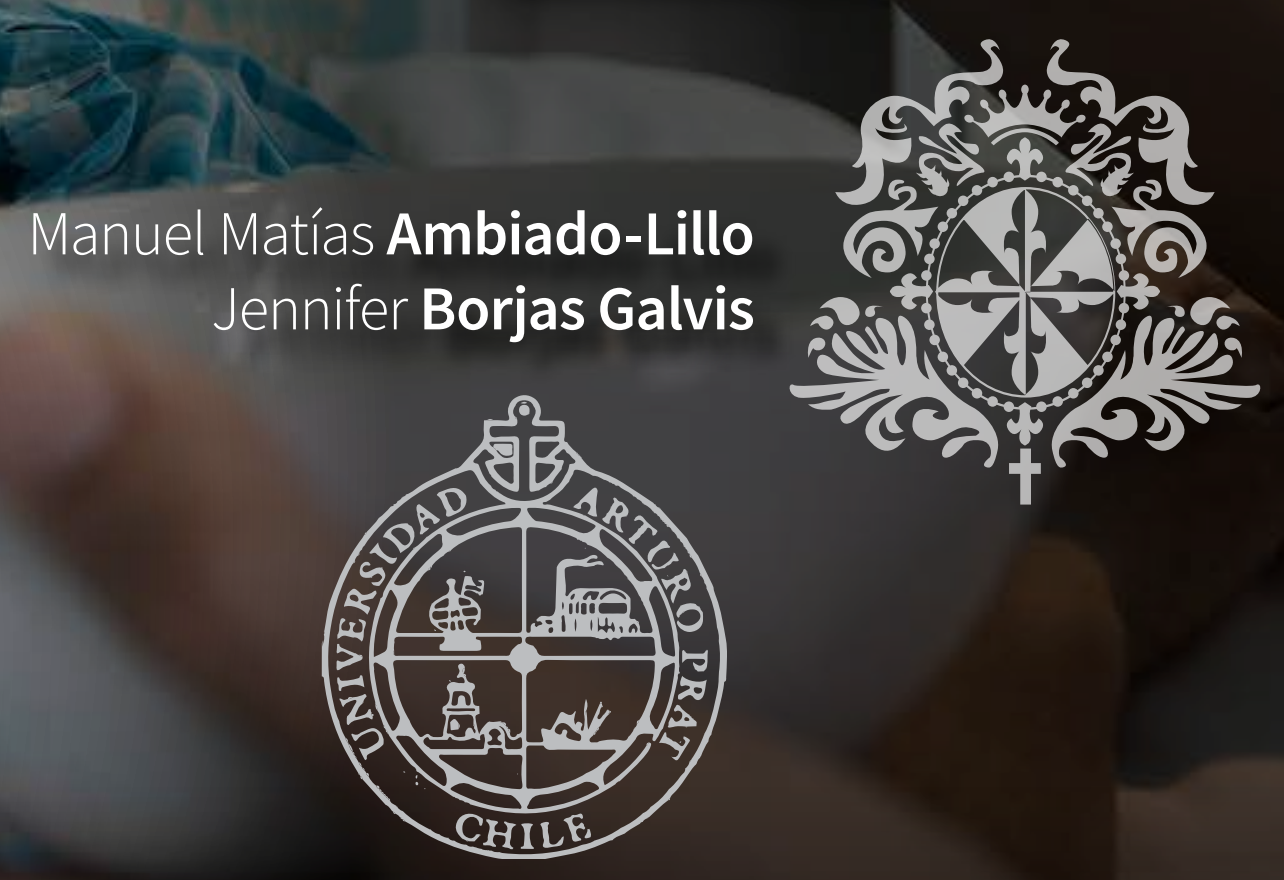


\section{ARETÉ \\ $1657-2513$}

ID:

10.33881/1657-2513.art.21110

Title: PresByphagia

Subtitle: A look at feeding and swallowing processes in older adults

Título: Presbifagia

Subtítulo: Una mirada a los procesos de alimentación y deglución en los adultos mayores

Alt Title / Título alternativo:

[en]: Presbifagia: Una mirada a los procesos de alimentación y deglución en los adultos mayores

[es]: Presbifagia: Una mirada a los procesos de alimentación y deglución en los adultos mayores

Author (s) / Autor (es):

Ambiado-Lillo, \& Borjas Galvis

Keywords / Palabras Clave:

[en]: Swallowing. Oropharyngeal

Dysphagia. Aging. Swallowing

neurophysiology. Presbyphagia.

Deglutition Disorders.. Swallowing Disorders.

[es]: Deglución. Disfagia Orofaríngea. Envejecimiento. Neurofisiología deglutoria. Presbifagia. Trastornos deglutorios.

Submited: 2021-05-12

Acepted: 2021-04-19

\section{Resumen}

Introducción: El proceso de envejecimiento, al igual que en todo el organismo, genera una serie de cambios en las estructuras estomatognáticas, y estos cambios morfológicos, característicos en el mecanismo de la deglución en sujetos sanos de edad avanzada caracterizan a la Presbifagia. Objetivo: Realizar un análisis crítico-reflexivo en torno a la conceptualización desde las diversas ópticas de los autores que exponen el fenómeno, con el fin de abrir la discusión dirigida a establecer un lenguaje unificado en pro de dirigir las acciones fonoaudiológicas hacia las áreas de promoción de la salud de la función deglutoria y prevención de la disfagia esta población. Metodología: Se seleccionaron, por conveniencia, artículos y capítulos de libros de especialidad publicados en la última década, que abordan las alteraciones deglutorias en adultos mayores, para comparar similitudes y diferencias en la forma de abordar el fenómeno. Resultados: Se evidencia que, pese al exponencial progreso de la disciplina, no existe

consenso conceptual entre los especialistas

al momento de hacer referencia a los cambios anatomofuncionales de la biomecánica deglutoria

y la transición de la presbifagia a la disfagia en los adultos mayores. Discusión: Una interesante discusión se genera en torno a la presbifagia y los parámetros de seguridad, efectividad, competencia y confortabilidad, dado que

los cambios neurológicos-estructurales son frecuentes en la tercera edad, los cuales acarrean diversas consecuencias, desde grados leves a severos, que impactan de forma diferenciada en los indicadores que habitualmente se describen en la clínica. Sin embargo, la conceptualización utilizada no es clara al realizar una distinción entre un estado transicional o de afección. Conclusión: Los cambios en el envejecimiento deben seguir siendo estudiados desde las diferentes concepciones para comprender mejor los fenómenos neurofisiológicos y anatómicos que se originan entorno a la Presbifagia.

\section{Citar como:}

Ambiado-Lillo, M. M., \& Borjas Galvis, J. (2021). Presbifagia: Una mirada a los procesos de alimentación y deglución en los adultos mayores. Revista Areté , 21 (1), 105-112. Obtenido de: arete.ibero.edu.co/article/view/2129

\section{Abstract}

Introduction: The aging process, as in the whole organism, generates a series of changes in the stomatognathic structures, and these morphological changes, characteristic of the swallowing mechanism in healthy elderly subjects, characterize Presbyphagia. Objective: To carry out a critical-reflective analysis around the conceptualization from the different perspectives

of the authors who expose the phenomenon, in order to open the discussion aimed at establishing

a unified language in favor of directing speech

therapy actions towards the areas of promotion of the health of the swallowing function and prevention of dysphagia in this population.

Methodology: Articles and chapters of specialty books published in the last decade, which address swallowing disorders in older adults, were selected

for convenience in order to compare similarities

and differences in the way of approaching the phenomenon. Results: It is evident that, despite the exponential progress of the discipline, there is no conceptual consensus among specialists when referring to anatomical and functional changes in swallowing biomechanics and the transition

from presbyophagia to dysphagia in older adults. Discussion: An interesting discussion is generated around presbyphagia and the parameters of

safety, effectiveness, competence and comfort, given that neurological-structural changes are

frequent in the elderly, which carry various consequences, from mild to severe degrees. that have a differentiated impact on the indicators that

are usually described in the clinic. However, the conceptualization used is not clear when making a distinction between a transitional state or a state

of affection. Conclusion: Changes in aging must continue to be studied from different conceptions to better understand the neurophysiological and anatomical phenomena that originate around Presbyphagia.
Manuel Matías Ambiado-Lillo, Msc

ORCID: $\quad$ 0000-0002-4653-3983

\section{Source I Filiacion:}

Universidad Arturo Prat

BIO:

Magíster en Neurociencias, Diplomado en Filosofía de la Ciencias Cognitivas. Área de experticia: Comunicación y Deglución en adultos y adultos mayores. Fonoaudiología. Facultad de Ciencias de la Salud. Universidad Arturo Prat. Iquique. Chile.

City I Ciudad:

Iquique (Chi)

e-mail:

mambiado@unap.cl
Jennifer Borjas Galvis, sp

ORCID: $\quad$ 0000-0003-0093-0665

\section{Source I Filiacion:}

Universidad del Rosario

BIO:

Fonoaudióloga - Universidad del Rosario. Esp. Administración en salud Pontificia Universidad Javeriana. Certificación Internacional Técnica NM-F Neuromuscular - Funcional Método Chiavaro - Candidata a Doctora en Fonoaudiología, Universidad del Museo Social Argentino, Buenos Aires Argentina. Área de experticia: Rehabilitación de deficiencias de la deglución en población infantil, adultos y adulto mayor..

City I Ciudad:

Bogotá ( Col) 


\section{Presbifagia Una mirada a los procesos de alimentación y deglución en los adultos mayores}

Presbyphagia: A look at feeding and swallowing processes in older adults Manuel Matías Ambiado-Lillo Jennifer Borjas Galvis

\section{Introducción}

Los procesos de alimentación y deglución han sido detalladamente descritos desde la perspectiva de ejecución neuromotora (Perlman, 1996), sin embargo, todo el procesamiento emotivo cognitivo solo ha tenido una creciente consideración en las últimas décadas. Como menciona (Paniagua, Rodríguez-Santos, \& Centera, 2019), comer es algo más que el simple hecho de tragar.

Para poder llevar a cabo una adecuada alimentación y deglución, es esencial que las estructuras centrales y periféricas estén extremadamente coordinadas entre sí, y a su vez, es imperioso que los centros reguladores deglutorios se presenten indemnes. De este modo se generan eventos de retroalimentación constante entre las estructuras fonoestomatológicas aferentes y eferentes, con los centros de integración-regulación situados a nivel cortical, subcortical y de tronco encefálico (Perlman, 1996); (Cámpora \& Falduti, 2019). Cuando todo este procesamiento y ejecución se realiza de forma coordinada, se evidencia una adecuada transición entre las cinco etapas clásicas de la deglución, a saber; anticipatoria, preparatoria oral, oral, faríngea y esofágica (Bielsa-Corrochano \& Urbasos-Garzón, 2019), no obstante, es importante mencionar que existen modelos de análisis biomecánicos que proponen una nueva conceptualización, para las etapas clásicas y los distintos tipos de consistencias que se degluten (Ortega, 2020). 


\section{Presbifagia}

Una mirada a los procesos de alimentación y deglución en los adultos mayores

Desde una perspectiva general, (Rosa \& Duek, 2018) proponen una visualización global de la alimentación y deglución en términos de efectividad y seguridad. La efectividad se entiende como la posibilidad de suplir la demanda hídrica y energética, que permite mantener la homeostasis corporal, mediante la ingesta de agua y alimentos; en tanto, la seguridad hace referencia a que estos eventos se lleven a cabo sin que generen penetraciones y/o aspiraciones antes, durante o después de deglutir. A estos dos conceptos se adicionan los de competencia, descrita como la adecuada ejecución y coordinación entre las estructuras estomatológicas implicadas en el proceso deglutorio; y la confortabilidad, relacionada con experimentar la alimentación como una experiencia placentera ( Bacco, Araya, Flores, \& Peña, 2014).

El proceso de envejecimiento, al igual que en todo el organismo, genera una serie de cambios en las estructuras estomatognáticas como se observa en la tabla 1 (Ferreira \& Ton, 2019); (NamasivayamMacdonald \& Riquelme, 2019). Estos cambios morfológicos, característicos en el mecanismo de la deglución en sujetos sanos de edad avanzada caracterizan a la presbifagia (Robbins, Hamilton, Lof , \& Kempster, 1992) citados por (Granell, Reyes, \& Campos, 2017).

\section{Tabla 1. Características deglutorias en presbifagia}

\begin{tabular}{|c|c|}
\hline Etapa de la deglución & Características \\
\hline Preparatoria Oral & $\begin{array}{c}\text { Alteraciones dentarias } \\
\text { Alteración del control motor oral del BA } \\
\text { Disminución sensorial gustativa } \\
\text { Alteración de la cohesión del BA } \\
\text { Déficits masticatorios } \\
\text { Escapes anteriores y posteriores }\end{array}$ \\
\hline Oral & $\begin{array}{l}\text { Presión lingual y fuerza reducida } \\
\text { Dificultad en la propulsión del BA }\end{array}$ \\
\hline Faríngea & $\begin{array}{c}\text { Retraso en el disparo de la respuesta motora } \\
\text { faríngea (RD) } \\
\text { Estasis de alimentos } \\
\text { Enlentecimiento del tránsito faríngeo } \\
\text { Disminución de la elevación laríngea } \\
\text { Enlentecimiento en la reconfiguración de la } \\
\text { encrucijada aerodigestiva } \\
\text { Acortamiento de la apnea deglutoria }\end{array}$ \\
\hline Esofágica & $\begin{array}{c}\text { Fallas en la distensión del EES } \\
\text { Disminución del peristaltismo esofágico } \\
\text { Reflujo } \\
\text { Fallas en la distensión del EEI }\end{array}$ \\
\hline
\end{tabular}

Fuente: Elaboración propia

Los parámetros de seguridad, efectividad, competencia y confortabilidad (SECC) pueden presentar variaciones o adaptaciones relacionadas con la etapa del ciclo vital y las comorbilidades adyacentes. Específicamente, en los adultos mayores de 65 años y más, se visualizan cambios orofaríngeos generados por el envejecimiento que impactan la neurofisiología y la anatomía. Dentro de las variaciones neurofisiológicas centrales y periféricos, como se exhibe en la tabla 2 (Robbins J., 1996), se observan modificaciones a nivel de la sustancia periventricular que ocasionan un efecto "ralentizador" que disminuye la velocidad de respuesta de los patrones neuromotores y sensitivos, que en asociación con los cambios en la configuración de las fibras musculares de la lengua disminuyen los valores de fuerza y presión máxima afectando el rendimiento de las fases preparatoria oral y oral en los subprocesos de captación, preparación y propulsión del bolo.
Tabla 2. Cambios centrales en la deglución asociados a presbifagia

\begin{tabular}{|c|c|}
\hline & $\begin{array}{c}\text { Cambios Neurofisiológicos Centrales y } \\
\text { Periféricos }\end{array}$ \\
\hline Cambios Periféricos & $\begin{array}{l}\text { Sarcopenia } \\
\begin{array}{l}\text { Disminución de la fuerza, el tono y la resistencia } \\
\text { muscular. }\end{array} \\
\text { Disminución progresiva en las unidades motoras } \\
\text { funcionales. } \\
\text { Aumento del tejido adiposo y conectivo de la lengua. }\end{array}$ \\
\hline Alteraciones centrales & $\begin{array}{l}\text { Hiperintensidades en la sustancia blanca y en los } \\
\text { Ganglios basales. } \\
\text { Aumento en el tiempo de respuesta y monitoreo } \\
\text { de las acciones por la disminución en la capacidad } \\
\text { para distinguir entre una señal eléctrica real y la } \\
\text { actividad aleatoria. } \\
\text { Desaceleración generalizada del rendimiento en las } \\
\text { tareas motoras finas y gruesas. } \\
\text { Disminución del tiempo de reacción a los estímulos } \\
\text { sensoriales (gusto, presión y temperatura). }\end{array}$ \\
\hline
\end{tabular}

Fuente: Elaboración propia

Así mismo, la fase faríngea, también presenta cambios asociados al envejecimiento, como la asincronía en el inicio de eventos laríngeos y faríngeos, incluido el cierre valvular del vestíbulo laríngeo, la excursión hiolaríngea máxima y la apertura del esfínter esofágico superior que se retrasa significativamente más tiempo en relación con el transporte de bolo oral que en adultos más jóvenes aumentando el riesgo de penetración laríngea, ocasiona que el bolo alimenticio permanezca un mayor tiempo en vallécula y senos piriformes incrementando el riesgo de aspiración (Ariza-Galindo \& Rojas Aguilar, 2020)

Como queda en evidencia, existe muchainformación especializada que aborda los cambios en la configuración de la biomecánica deglutoria en los adultos mayores, pese a ello, no se identifican propuestas teóricas que permitan la delimitación conceptual unificada del concepto de presbifagia.

\section{Objetivo general}

Realizar un análisis crítico-reflexivo en torno a la conceptualización desde las diversas perspectivas de los autores que exponen el fenómeno, con el fin de abrir la discusión dirigida a establecer un lenguaje unificado en pro de dirigir las acciones fonoaudiológicas hacia las áreas de promoción de la salud de la función deglutoria y prevención de la disfagia esta población.

\section{Método}

Para realizar el análisis crítico-reflexivo se seleccionaron, por conveniencia, artículos y capítulos de libros de especialidad publicados en las últimas décadas, que abordan las alteraciones deglutorias en adultos mayores, para comparar similitudes y diferencias en la forma de abordar el fenómeno.

Para la selección de información se realizaron dos búsquedas diferenciadas. La primera de ellas consideró la revisión de literatura, específicamente ligada a alteraciones deglutorias en adultos mayores, proveniente de revistas científicas indexadas en "Web of Science", "Scopus" y "Scielo", contemplando artículos desde el 2007 a la actualidad. La segunda, se extendió hacia las bases neurofisioanatómicas implicadas en los procesos deglutorios, tomando como referencia los mismos motores de búsqueda. Ambas 
indagaciones se complementaron con la inclusión de información procedente de libros del área de especialidad publicados en los últimos 5 años.

\section{Resultados}

Se evidencia que pese al exponencial progreso de la disciplina, no existe consenso conceptual entre los especialistas al momento de hacer referencia a los cambios anatomofuncionales de la biomecánica deglutoria y la transición de la presbifagia a la disfagia en los adultos mayores. Sin embargo, un esfuerzo por aunar las visiones podría encontrar su punto de conciliación en los parámetros SECC, los cuales se exponen relacionados con la presbifagia a continuación, y según los autores, dan pie para la apertura del diálogo de cara a la discusión.

\section{Presbifagia y parámetros SECC}

Una interesante discusión se genera en torno a la presbifagia y los parámetros SECC. Como se ha descrito previamente, los cambios neurológicos-estructurales son frecuentes en la tercera edad, y como es de esperar, estos cambios acarrean diversas consecuencias, desde grados leves a severos, que impactan de forma diferenciada en los parámetros que habitualmente se describen en la clínica.

Para Rosa y Duek (2018), los cambios que se presentan repercuten de forma directa en la competencia y la confortabilidad de las personas; en la misma línea, para (Ortega \& Clavé, 2017) citado por (Venegas, Navia, Fuentealba, Diez de Medina, \& Kunstmannaa , 2020) el parámetro de seguridad no se ve alterado. En oposición, para (NamasivayamMacdonald \& Riquelme, 2019), todos los cambios tienen un impacto directo en la seguridad.

Para esclarecer la divergencia que existe en las diversas visiones se puede realizar un análisis general desde la neurofisiología. Al partir de los cambios neurológicos-estructurales, se puede establecer que en mayor o menor medida, la coordinación necesaria para ejecutar el proceso deglutorio se altera derivando en alteraciones de competencia, las que a su vez, tienen un impacto directo en los mecanismos de protección aferentes y/o eferentes, sin embargo, las capacidades preservadas de las personas podrían enmascarar los posibles déficits en sus formas leves, generando dificultades en su identificación (Ferrero López, García Gollarte, Botella, \& Vidal, 2012). Un par de ejemplos que permiten ilustrar esta relación son, (1) incompetencia para generar un adecuado contacto entre el dorso de la lengua y el velo, lo que podría generar un escape posterior y posibilidad de penetraciones pre-deglutorias, (2) incompetencia en la coordinación de las aferencias orofaríngeas, posibilitando un aumento en la latencia de los mecanismos de protección, o (3) incompetencia en el ascenso y adelantamiento del complejo hiolaríngeo, favoreciendo penetraciones post-deglutorias.

En cuanto al parámetro de confortabilidad, la pérdida de la sensación placentera para alimentarse puede derivar en un estado de inapetencia o hiperoxia, el cual sostenido en el tiempo puede impactar directa e inevitablemente en la efectividad, dado que, al disminuir la frecuencia de los periodos de alimentación, o la cantidad de alimentos consumidos en cada tiempo, disminuirá la ingesta calórica que, a su vez, reduce la reserva funcional en el adulto mayor ocasionando posibles desequilibrios en la homeostasis corporal que incrementan el riesgo de alteración en la función deglutoria.

\section{Discusión}

\section{Presbifagia, ¿afección o estado de transición?}

En la actualidad, la presbifagia se entiende como un estado transicional entre el proceso deglutorio saludable y la disfagia orofaríngea producto de todos los cambios anátomofuncionales, sean estos centrales y/o periféricos. Aparentemente, un criterio clave que marca la diferencia por exclusión en la conceptualización, es la alteración en el parámetro de seguridad.

Estudios objetivos sustentan un aumento de residuo con la edad, posiblemente relacionado con una limitada apertura del esfínter esofágico superior (UES) en adultos mayores (Cook, y otros, 1994). Los adultos mayores con un envejecimiento saludable, pueden experimentar episodios de penetración asociados a los cambios descritos en cada una de las etapas deglutorias, siendo el residuo faríngeo el principal signo en videofluoroscopia de cambios en la fase faríngea (Ariza-Galindo \& Rojas Aguilar, 2020).

Un cuestionamiento que surge desde esta postura clásica es si la presbifagia se debe considerar como disfagia orofaríngea, o si los cambios fisiológicos corresponden a las adaptaciones requeridas para compensar el efecto de una deglución "ralentizada" asociada al envejecimiento. Al realizar un análisis sobre la fisiología del proceso deglutorio se puede establecer que cualquier alteración en las etapas de formación y traslado del bolo alimenticio al estómago estaría enmarcada dentro deestaentidad nosológica, portanto, las alteraciones en etapas anticipatorias y preparatorias que tributan en gran medida al parámetro de confortabilidad serían signos claros de alteración de la función deglutoria. Mientras que, si se incluye en el análisis la dimensión cognitiva-emotiva, podría alinearse con la sensación de inapetencia derivada de las alteraciones de confortabilidad y competencia

Otros autores, formulan que la presbifagia se caracteriza por una función de deglución que, aunque conservada, puede presentar sutiles signos de deterioro, que suelen ser asintomático pero que tienden a empeorar lentamente a medida que avanza el proceso de envejecimiento, el cual no compromete las primeras fases de deglución, pero si aumenta el riesgo de desarrollar una deglución disfuncional o disfagia (Humbert \& Robbins, 2008).

Por otro lado, un comité de miembros de la Sociedad Europea de Desórdenes de la Deglución y la Sociedad de Medicina Geriátrica de la Unión Europea, se refiere a disfagia orofaríngea (DO), como las alteraciones a nivel del tránsito de alimentos o líquidos deficiente o incómodo desde la cavidad bucal hasta el esófago, la cual puede afectar de manera grave y frecuente a todos los fenotipos de los pacientes mayores, ya que la respuesta de deglución orofaríngea está alterada en las personas mayores y puede causar aspiración (Baijens, y otros, 2016). Adicionalmente, se refieren a estos desordenes como un "Síndrome Geriátrico" que representa una alta prevalencia entre las personas mayores, causada por múltiples factores, asociados a comorbilidades y mal pronóstico, que necesita un enfoque multidimensional para ser tratado.

Los autores creen que el debate central en la actualidad debería orientarse a determinar si efectivamente la alteración en seguridad es el hito que determina el paso de la presbifagia a la disfagia orofaríngea, entendiéndose como un estado transicional, o por el contrario, "es" una disfagia asintomática que se identifica a posteriori, dado que 


\section{Presbifagia}

Una mirada a los procesos de alimentación y deglución en los adultos mayores

el envejecimiento es un factor de riesgo para la disminución de la respuesta inmunológica y para la reducción general de la reserva funcional (Santos, Ribeiro, Jesús, \& Matos, 2021), lo que ratificaría la dificultad para el diagnóstico clínico en estados iniciales, el cual solo se haría evidente mediante la manifestación de signos y síntomas frecuentes como: dificultades en la función masticatoria con sólidos, tos post deglución, acumulación o disminución de saliva, residuos en cavidad oral y faríngea, deshidratación y baja de peso, entre otros.

Pero independiente de cuál sea la clasificación utilizada, el panorama es claro en cuanto a la necesidad de redirigir el rol fonoaudiológico hacia acciones de promoción de la salud deglutoria en el adulto mayor y a la prevención de la disfagia a fin de detectar estas dificultades de manera temprana minimizado el riesgo de estadios crónicos que deterioren la calidad de vida de los adultos mayores. Dentro de las herramientas de promoción de la salud deglutoria, se pueden sumar estrategias como la higiene oral, que puede favorecer en primer lugar a reducir la colonización de bacterias que durante los episodios de penetración/ aspiración con saliva puedan producir afecciones respiratoria asociadas con neumonías. Algunos estudios recomiendan evaluación sistemática de la salud bucal en pacientes ancianos con disfagia orofaríngea, ya que refieren que estos presentan múltiples morbilidades y deterioro del estado de salud, alta prevalencia de signos de deterioro de la seguridad de la deglución y mal estado de salud bucal con alta prevalencia de enfermedades periodontales y caries que aumentan el riesgo de desarrollar neumonías aspirativa (Ortega, Parra, Zarcero, Nart, Sakwinska, \& Clavé, 2014).

Adicionalmente, cabe dentro de las estrategias promover una adecuada adaptación de prótesis dentales que faciliten la función masticatoria, garantizando hábitos de alimentación saludable en las etapas preparatoria oral y oral para la ingesta de sólidos. A medida que se envejece, en la cavidad oral se producen cambios estructurales, por lo que adultos y adultos mayores requieren del uso de prótesis dental que en ocasiones puede resultar insatisfactoria y ocasionar cambios en los hábitos alimenticios, sin embargo, plantean que la calidad de vida se relaciona con los aspectos de salud oral y satisfacción del usuarios de prótesis dental (Farias-Neto, y otros, 2012) y (Michaud, de Grandmont, Feine, \& Emami, 2012) citados en (Moya, Marquardt, Arellano, Contreras, \& González, 2019), por lo cual se hace relevante el seguimiento y trabajo interdisciplinario en este aspecto.

Así mismo, dentro de las estrategias de prevención dirigidas a la detección temprana de los signos y síntomas que puedan representar un factor de riesgo observable mediante los parámetros SECC en el adulto mayor, se sugieren la implementación de cuestionarios de autopercepción que faciliten la identificación de los signos y síntomas asociados a la disfagia, como un predictor que a priori oriente al clínico hacia la implementación de test de cribado y/o protocolos de evaluación en los adultos mayores que se encuentren en el periodo de transición de los primeros signos de disfagia orofaríngea, de manera que aún se puedan tomar acciones tempranas como, modificaciones en la dieta y cambios posturales que impacten positivamente la fisiología deglutoria.

El test de autopercepción más utilizado es el Test Eating Assessment Tool EAT-10 (Giraldo-Cadavid, y otros, 2016); (FernándezRosati, Lera, Fuentes-López, \& Albala, 2018), considerada una herramienta de autoevaluación válida y sólida capaz de medir el riesgo de disfagia e identificar a las personas que necesitan una intervención multidisciplinaria, sin embargo, se recomienda una lectura crítica del artículo "Evaluating the Psychometric Properties of the Eating Assessment Tool (EAT-10) Using Rasch Analysis", en el cual se expone que la prueba presenta debilidades significativas en la validez estructural y la consistencia interna (Cordier, y otros, 2017).
Otros test de detección temprana son: Toronto Test (TOR-BSST), descrito como un método relativamente rápido y fácil de usar para evaluar la ingestión de agua (Martino, y otros, 2009) el Test Gugging Swallowing Screen (GUSS) (Trapl, y otros, 2007) es otra prueba de detección de cabecera simple que permite la evaluación de la función de deglución indirecta y directamente a través de consistencias semisólidas, líquidas y sólidas; el método de exploración clínica volumen-viscosidad (MECV-V) que se basa en la administración secuencial de bolos en viscosidades néctar, líquido y pudding con volúmenes crecientes a medida que progresa la evaluación (Clavé, Arreola, Romea, Medina, Palomera, \& Serra-Prat, 2008); y por último, un test recientemente estudiado para incluir en el proceso de evaluación la habilidad masticatoria, el Test de Deglución y Masticación de Sólidos (TOMASS) (Huckabee, y otros, 2018), propuesto para la medición de 4 parámetros cuantitativos: número de ciclos masticatorios, numero de bocados, numero de degluciones, y tiempo total de deglución por 1/4 de galleta salada, aplicado en pacientes adultos mayores que tengan piezas dentales y/o prótesis dentales bien adaptadas.

Dentro de la evaluación instrumental de la deglución, se incluyen exámenes objetivos con mayores tasas de sensibilidad y especificidad, relevantes en la detección de los cambios transicionales en el adulto mayor que no serán identificados mediante la evaluación clínica, como las aspiraciones silentes, dadas por los cambios aferentes a nivel faríngeo y laringotraqueal. Dentro de estos, se incluyen principalmente la videofluroscopia (VF) de la deglución que permite observar de forma dinámica los eventos biomecánicos en cada etapa, mientas que la video endoscopía (FEES), contribuye a la observación directa de la región faringolaríngea.

Procedimientos de intervención no se discutirán en el presente artículo dado que escapan a su objetivo final. No obstante, se recomienda la lectura de las publicaciones de (Gutiérrez-Achury, RualesSuárez, Giraldo-Cadavid, \& Rengifo-Varona, 2015); (Higashiguchi, y otros, 2018); (Field, Wenke, Sabet, Lawrie, \& Cardell, 2018); (NamasivayamMacdonald \& Riquelme, 2019) y (Zimmerman, Carnaby, Lazarus, \& Maldandraki, 2020) quienes profundizan en su análisis.

\section{Conclusiones}

Los cambios en el envejecimiento deben seguir siendo estudiados desde las diferentes concepciones para comprender mejor los fenómenos neurofisiológicos y anatómicos que se originan entorno a la presbifagia, lo que permitirá perfeccionar los mecanismos de detección temprana que anticipen un deterioro mayor en la función deglutoria.

El fonoaudiólogo, tiene un papel principal dentro de los equipos interdisciplinarios en esta población que cada vez se hace más creciente, ejerciendo actividades en los dominios de: colaboración, consejería, prevención y promoción, detección temprana, propuestos por la ASHA, lo que implica redirigir las acciones desde un enfoque de rehabilitación hacia uno de atención primaria en salud (APS), basado en funciones de promoción y prevención de la "Salud Deglutoria en el Adulto Mayor", favoreciendo así el bienestar y el mantenimiento de la calidad de vida en esta población.

En la actualidad no se cuenta con una descripción unificada y clara de lo que es la presbifagia, por este motivo, los autores pretenden que el presente manuscrito sea un aporte a la reflexión y delimitación conceptual dentro del área de especialidad. 


\section{Referencias}

Ariza-Galindo, C., \& Rojas Aguilar, D. (2020). Disfagia en el adulto mayor. Universitas Médica, 61(4), 117-128. https://doi.org/10.11144/javeriana. umed61-4.disf

Bacco, J., Araya, F., Flores, E., \& Peña, N. (2014). Trastornos de la alimentación y deglución en niños y jóvenes portadores de parálisis cerebral: abordaje multidisciplinario. Revista Médica Clínica Las Condes, 25(2), 330-342. https://doi.org/10.1016/S0716-8640(14)70044-6

Baijens, L., Clavé, P., Cras, P., Ekberg, O., Forster, A., Kolb, G., y otros. (2016). European society for swallowing disorders - European Union Geriatric Medicine Society white paper: oropharyngeal dysphagia as a geriatric syndrome. Clinical Interventions in Aging, 11, 1403-1428.

Bielsa-Corrochano, M., \& Urbasos-Garzón, C. (2019). Anatomofisiología de la deglución. En Paniagua, Jaime, Susanibar, Franklin, P. Giménez, P. Murciego, \& R. García, Disfagia. De la evidencia científica a la práctica clínica. Madrid: Editorial GiuntiEOS.

Broyles, L., Tate, J., \& Happ, M. (2012). Use of augmentative and alternative communication strategies by family members in the intensive care unit. Am J Crit Care. Mar. 21(2): e21-32. doi: https://doi.org/10.4037/ ajcc2012752. PMID: 22381993 [PubMed - indexed for MEDLI.

Cámpora, H., \& Falduti, A. (2012). Evaluación y tratamiento de las alteraciones de la deglución. Rev Am Med Resp, 3(1), 98-107.

Cámpora, H., \& Falduti, A. (2019). Deglución de la A a la Z. Fisiopatología, Evaluación, Tratamiento. Buenos Aires, Argentina: Ediciones Journal.

Clavé, P., Arreola, V., Romea, M., Medina, L., Palomera, E., \& Serra-Prat, M. (2008). Accuracy of the volume-viscosity swallow test for clinical screeneng of oropharyngeal dysphagia and aspiration. Clinical Nutrition, 2(7), 806-15. https://doi.org/10.1016/j.clnu.2008.06.011

Cook, I., Weltman, M., Wallace, K., Shaw, D., McKay, E., Smart, R., y otros. (1994). Influence of aging on oral-pharyngeal bolus transit and clearance during swallowing: Scintigraphic study. American Journal of Physiology - Gastrointestinal and Liver Physiology, 266(6), 972-977. https://doi.org/10.1152/ajpgi.1994.266.6.9972

Cordier, R., Joosten, A., Clavé, P., Schindler, A., Demir, N., Arslan, S., y otros. (2017). Evaluating the Psychometric Properties of the Eating Assessment Tool (EAT-10) Using Rasch Analysis. Dysphagia, 32(2), 250-260. https://doi.org/10.1007/s00455-016-9754-2

Farias-Neto, A., Pereira, B., Xitara, R., Germano, A., Ribeiro, J., MestrinerJunior, W., y otros. (2012). The influence of mandibular implantretained overdentures in masticatory efficiency. Gerodontology, 29(2), 650-655.

Fernández-Rosati, J., Lera, L., Fuentes-López, E., \& Albala, C. (2018). Validez y confiabilidad del cuestionario Eating Assessment Tool 10 (EAT10) para detectar disfagia en adultos mayores chilenos. Revista Médica de Chile, 146(9), 1008-1015. https://doi.org/10.4067/s003498872018000901008

Ferreira, T., \& Ton, V. (2019). Herramientas clínicas en el abordaje de la presbifagia. En J. Paniagua, F. Susanibar, P. Giménez, P. Murciego, \& R. García, Disfagia. De la evidencia científica a la práctica clínica. EOS. Madrid: Editorial GiuntiEOS.

Ferrero López, M., García Gollarte, J., Botella, J., \& Vidal, O. (2012). Detección de disfagia en mayores institucionalizados. Revista Espanola de Geriatria y Gerontologia, 47(4), 143-147. https://doi.org/10.1016/j. regg.2011.09.004

Field, M., Wenke, R., Sabet, A., Lawrie, M., \& Cardell, E. (2018). Implementing Cough Reflex Testing in a Clinical Pathway for Acute Stroke: A Pragmatic Randomised Controlled Trial. Dysphagia, 33(6), 827-839. https://doi.org/10.1007/s00455-018-9908-5

Giraldo-Cadavid, L., Gutiérrez-Achury, A., Ruales-Suárez, K., Rengifo-Varona, M., Barros, C., Posada, A., y otros. (2016). Validation of the Spanish Version of the Eating Assessment Tool-10 (EAT-10spa) in Colombia. Blinded Prospective Cohort Study. Dysphagia, 31(3), 398-406. https:// doi.org/10.1007/s00455-016-9690-1
Granell, J., Reyes, P., \& Campos, V. (2017). Etiopatogenia de los trastornos de la deglución. En R. Gutierrez, I. Méndez, L. Garrido, J. Granell, P. Reyes, V. Campos, y otros, Disfagia Orofaríngea: Conceptos y conocimientos necesarios para el especialista de ORL (Vol. 8, págs. 16-22). Madrid, Madrid: Asociación Madrileña de Otorrinolaringología (AMORL)

Gutiérrez-Achury, A., Ruales-Suárez, K., Giraldo-Cadavid, L., \& RengifoVarona. (2015). Escalas de calidad de vida y valoración de los síntomas en disfagia. Revista Med, 23(1), 52-57. https://doi. org/10.18359/rmed.1329

Higashiguchi, T., Ohara, H., Kamakura, Y., Kikutani, T., Kuzuya, M., Enoki, H., y otros. (2018). Efficacy of a New Post-Mouthwash Intervention (Wiping Plus Oral Nutritional Supplements) for Preventing Aspiration Pneumonia in Elderly People: A Multicenter, Randomized, Comparative Trial. Annals of Nutrition and Metabolism, 71(3-4), 253-260. https://doi.org/10.1159/000485044

Huckabee, M., McIntosh, T., Fuller, L., Curry, M., Thomas, P., Walshe, M., y otros. (2018). The Test of Masticating and Swallowing Solids (TOMASS): reliability, validity reliability and international normative data. . International Journal of Language and Communication Disorders, 53(1), 144-156. https://doi.org/10.1111/1460-6984.12332

Humbert, I., \& Robbins, J. (2008). Dysphagia in the Elderly. Physical Medicine and Rehabilitation Clinics of North America,, 4, 853-866. https://doi.org/10.1016/j.pmr.2008.06.002

Martino, R., Silver, F., Teasell, R., Bayley, M., Nicholson, G., Streiner, D., y otros. (2009). The toronto bedside swallowing screening test (TORBSST) development and validation of a dysphagia screening tool for patients with stroke. Stroke, 40(2), 555-561. https://doi.org/10.1161/ STROKEAHA.107.510370

Moya, M., Marquardt, K., Arellano, C., Contreras, C., \& González, C. (2019). Efectos de la Prótesis Dental en la Función Masticatoria de Adultos. Journal of Health Medical Science, 5(1), 41-50.

Namasivayam-Macdonald, A., \& Riquelme, L. (2019). Presbyphagia to dysphagia: Multiple perspectives and strategies for quality care of older adults. Seminars in Speech and Language, 40(3), 227-242. https://doi.org/10.1055/s-0039-1688837

Ortega, F. (2020). Disfagia Neurogénica. Bases Conceptuales y Metodologicas para la Intervención Fonoaudiológica de Trastornos de la Deglución. Santiago de Chile: ENM editions.

Ortega, O., \& Clavé, P. (2017). Diagnosis and Management of oropharyngeal dysphagia among older persons, State of Art. Review Article. Journal of Post-Acute and Long Term Care Medicine (JAMDA), 576-582.

Ortega, O., Parra, C., Zarcero, S., Nart, J., Sakwinska, O., \& Clavé, P. (2014). Oral health in older patients with oropharyngeal dysphagia. Age and Ageing, 43(1), 132-137. https://doi.org/10.1093/ageing/aft164

Paniagua, J., Rodríguez-Santos, F., \& Centera, M. (2019). Comer, algo más que tragar. En J. Paniagua, F. Susanibar, P. Giménez, P. Murciego, \& R. García, Disfagia. De la evidencia científica a la práctica clínica. Madrid: Editorial GiuntiEOS.

Perlman, A. (1996). Dysphagia in Stroke Patients. Seminars in Neurology, 16(4), 341-348. https://doi.org/10.1055/s-2008-1040992

Robbins , J., Hamilton, J., Lof , G., \& Kempster, G. (1992). Oropharyngeal swallowing in normal adults of different ages. Gastroenterology, 103, 823-829.

Robbins, J. (1996). Normal Swallowing and Aging. Seminars in Neurology, 16(4), 309-317. https://doi.org/10.1055/s-2008-1040989

Rosa, I., \& Duek, V. (2018). Envejecimiento y Deglución. En M. Trovato, I. Rosa, \& G. Brotzman, Manual de fonoestomatología. Clínica, diagnóstico y tratamiento de las alteraciones en la deglución y alimentación en niños y adultos. Buenos Aires: Akadia.

Santos, J., Ribeiro, O., Jesús, L., \& Matos, M. (2021). Interventions to prevent aspiration pneumonia in older adults: An updated systematic review. Journal of Speech, Language, and Hearing Research, 64(2), 464-480. https://doi.org/10.1044/2020 JSLHR-20-00123 


\section{Presbifagia}

Una mirada a los procesos de alimentación y deglución en los adultos mayores

Trapl, M., Enderle, P., Nowotny, M., Teuschl, Y., Matz, K., Dachenhausen, A., y otros. (2007). Dysphagia bedside screening for acute-stroke patients: The gugging swallowing screen. Stroke, 38(11), 2948-2952. https://doi. org/10.1161/STROKEAHA.107.483933

Venegas, M., Navia, R., Fuentealba, I., Diez de Medina, M., \& Kunstmannaa , P. (2020). Manejo hospitalario de la persona mayor con disfagia. Revista Médica Clínica Las Conde, 31(1), 50-64. https://doi. org/10.1016/j.rmclc.2019.09.005

Zimmerman, E., Carnaby, G., Lazarus, C., \& Maldandraki, G. (2020). Motor Learning, Neuroplasticity, and Strength and Skill Training: Moving From Compensation to Retraining in Behavioral Management of Dysphagia. American Journal of Speech-Language Pathology, 29(2S), 1065-1077. https://doi.org/10.1044/2019 AJSLP-19-00088

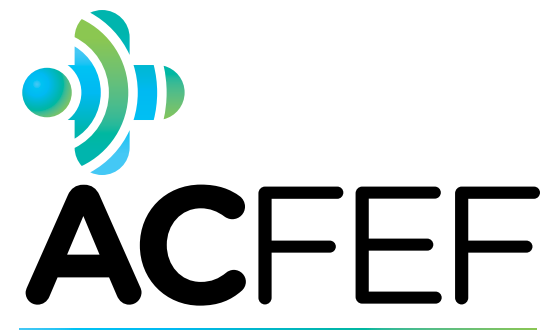

Atención Clínica y Formación Especializada en Fonoaudiología 